\title{
The effect of nanowire length and diameter on the properties of transparent, conducting nanowire films $\dagger$
}

\author{
Stephen M. Bergin, ${ }^{a}$ Yu-Hui Chen, ${ }^{b}$ Aaron R. Rathmell, ${ }^{a}$ Patrick Charbonneau, ${ }^{a}$ Zhi-Yuan Li ${ }^{b}$ \\ and Benjamin $\mathrm{J}$. Wiley ${ }^{*} a$
}

Received 14th January 2012, Accepted 6th February 2012
DOI: 10.1039/c2nr30126a

This article describes how the dimensions of nanowires affect the transmittance and sheet resistance of a random nanowire network. Silver nanowires with independently controlled lengths and diameters were synthesized with a gram-scale polyol synthesis by controlling the reaction temperature and time. Characterization of films composed of nanowires of different lengths but the same diameter enabled the quantification of the effect of length on the conductance and transmittance of silver nanowire films. Finite-difference time-domain calculations were used to determine the effect of nanowire diameter, overlap, and hole size on the transmittance of a nanowire network. For individual nanowires with diameters greater than $50 \mathrm{~nm}$, increasing diameter increases the electrical conductance to optical extinction ratio, but the opposite is true for nanowires with diameters less than this size. Calculations and experimental data show that for a random network of nanowires, decreasing nanowire diameter increases the number density of nanowires at a given transmittance, leading to improved connectivity and conductivity at high transmittance $(>90 \%)$. This information will facilitate the design of transparent, conducting nanowire films for flexible displays, organic light emitting diodes and thin-film solar cells.

Indium tin oxide (ITO) is the material of choice for transparent conducting films in flat-panel displays, organic solar cells, and organic light emitting diodes because, with a sheet resistance of $10 \Omega \mathrm{sq}^{-1}$ at a transmittance of $90 \%(\lambda=550 \mathrm{~nm})$, it is highly conductive and transparent. However, indium is a scarce element, ITO is brittle, and ITO film is expensive because it is produced with a vapor-phase coating process that is 1000 times slower than newspaper printing. ${ }^{1,2}$ These problems have motivated a search for alternatives to ITO that are flexible and can be deposited from liquids at high coating rates. ${ }^{3-9}$ As discussed in recent reviews, promising solution-processed alternatives to ITO include poly(3,4-ethylenedioxythiophene)poly(styrenesulfonate), carbon nanotubes, graphene, ITO nanowires, and metal nanowires. ${ }^{10-15}$ Of these alternatives, films of silver nanowires currently have the highest conductance and transmittance. ${ }^{4,16-18}$ For example, Leem et al. have recently reported obtaining silver nanowire films with a sheet resistance of $10 \Omega \mathrm{sq}^{-1}$ at a transmittance of $89.3 \%(\lambda=550 \mathrm{~nm})$, nearly matching ITO. Although silver $\left(\$ 1000 \mathrm{~kg}^{-1}\right)$ is more expensive than indium $\left(\$ 800 \mathrm{~kg}^{-1}\right)$, the

${ }^{a}$ Department of Chemistry, Duke University, 124 Science Dr, Box 90354, Durham, NC 27708, USA. E-mail: benjamin.wiley@duke.edu

${ }^{b}$ Laboratory of Optical Physics, Institute of Physics, Chinese Academy of Sciences, Beijing 100190, P. R. China

$\dagger$ Electronic supplementary information (ESI) available: Includes methods and transmission spectra of nanowire films. See DOI: $10.1039 / \mathrm{c} 2 \mathrm{nr} 30126 \mathrm{a}$ fact that silver nanowire films can be produced with highthroughput wet-coating processes allows them to achieve lower costs. ${ }^{19,20}$

Here we report a simple polyol synthesis that enables control over the length and diameter of silver nanowires, as well as their production on the gram scale. By measuring the properties of films composed of nanowires with distinct ranges of dimensions, we have obtained the first quantitative confirmation of theoretical predictions for the effect of nanowire length and number density on the conductance of 2D nanowire networks. In addition, the transmittance of a nanowire network is shown to be inversely proportional to the area coverage of nanowires. Finally, with a combination of simulations and experimental measurements, we explore the subtle ways in which nanowire diameter affects the optoelectronic performance of nanowire networks.

\section{Results and discussion}

Production of silver nanowires with controlled dimensions

To synthesize silver nanowires, we used a polyol synthesis that has been scaled-up and simplified compared to those that have previously been reported. ${ }^{21}$ To start, $158.4 \mathrm{ml}$ of J.T. Baker ethylene glycol (EG) was added to a $500 \mathrm{ml}$ flask. This flask was stoppered and placed in an oil bath set to the desired temperature. We highly recommend the use of J.T. Baker EG because 
of its low iron and chloride content; these ions have a dramatic effect on the reaction product. ${ }^{21}$ Four solutions were then prepared: (1) $0.257 \mathrm{~g}$ of $\mathrm{NaCl}$ in $20 \mathrm{ml} \mathrm{EG}$, (2) $0.081 \mathrm{~g} \mathrm{Fe}\left(\mathrm{NO}_{3}\right)_{3}$ in $10 \mathrm{ml} \mathrm{EG}$, (3) $1.05 \mathrm{~g}$ polyvinylpyrrolidone (55,000 MW) in 25 $\mathrm{ml} \mathrm{EG}$, and (4) $1.05 \mathrm{~g} \mathrm{AgNO}_{3}$ in $25 \mathrm{ml} \mathrm{EG}$. After preheating the EG in an oil bath for $1 \mathrm{~h}, 0.2 \mathrm{ml}$ of solution (1), $0.1 \mathrm{ml}$ of solution (2), $20.76 \mathrm{ml}$ of solution (3), and $20.76 \mathrm{ml}$ of solution (4) were added to the flask in that order with a single addition from a pipette, with about $30 \mathrm{~s}$ between the addition of each solution (the time between additions is not critical). The flask was stoppered and allowed to react for a given time. In comparison to previous reports, this synthesis has been scaled up by ten times, requires no controlled addition with a syringe pump, and allows for the production of nanowires over a range of different sizes. ${ }^{21}$

Previous work has shown that the reducing power of EG increases with temperature, and that this effect is due to the temperature-dependent oxidation of ethylene glycol to glycolaldehyde, the reducing agent. ${ }^{22}$ The temperature-dependent reducing power of EG is apparent in Fig. 1A, which clearly shows nanowire growth occurs more rapidly at higher temperatures. For example, nanowires grew to a length of $5 \mu \mathrm{m}$ at $160{ }^{\circ} \mathrm{C}$ within $30 \mathrm{~min}$, but it took two hours for them to reach the same length at a reaction temperature of $140^{\circ} \mathrm{C}$. More importantly for this work, Fig. 1A \& B illustrate that longer nanowires with larger diameters could be produced at lower reaction temperatures. For example, the longest nanowires that could be obtained at $160{ }^{\circ} \mathrm{C}$ were $7.5 \mu \mathrm{m}$ in length and $45 \mathrm{~nm}$ in diameter, while those produced at $130^{\circ} \mathrm{C}$ could reach lengths of over $20 \mu \mathrm{m}$, with diameters of $65 \mathrm{~nm}$.

We hypothesized that the reason the nanowires grew longer and wider at lower temperatures was because the nucleation rate was lower at lower reaction temperatures, leaving more silver precursor per nuclei. To test this hypothesis, we measured and plotted the percent conversion of $\mathrm{Ag}^{+}$to $\mathrm{Ag}^{0}$ vs. reaction time (Fig. 1C). This plot confirms the reduction rate was greater at higher reaction temperatures, which in turn likely supported a higher nucleation rate. Not surprisingly, the time at which the maximum percent conversion was reached roughly corresponds to the time at which nanowires stopped growing. Given that both high and low temperature reactions produced roughly the same mass of reduced silver, but wires of different lengths, we conclude that the higher temperature reactions contained a higher number density of nanowires, and thus a higher density of nanowire nuclei (five-fold twinned decahedra) must have formed during the nucleation stage of the reaction. ${ }^{23,24}$

The decrease in the percent conversion seen at the very end of the high-temperature reactions could be due to settling and aggregation of the wires, as well as etching by nitric acid. Acid is generated during the reduction of silver ions by glycolaldehyde through eqn (1). The greater rate of reduction at higher temperatures

$$
\begin{aligned}
\mathrm{HOCH}_{2} \mathrm{CHO}+ & 2 \mathrm{Ag}^{+}+\mathrm{H}_{2} \mathrm{O} \rightarrow \mathrm{HOCH}_{2} \mathrm{COOH} \\
& +2 \mathrm{Ag}^{0}+2 \mathrm{H}^{+}
\end{aligned}
$$

likely resulted in a more acidic environment, and thus a greater etching rate. Using a $\mathrm{pH}$ strip, we measured the $160{ }^{\circ} \mathrm{C}$ reaction to have a $\mathrm{pH}=4$ at $30 \mathrm{~min}$, and $\mathrm{pH}=2$ at $2 \mathrm{~h}$. The reaction also
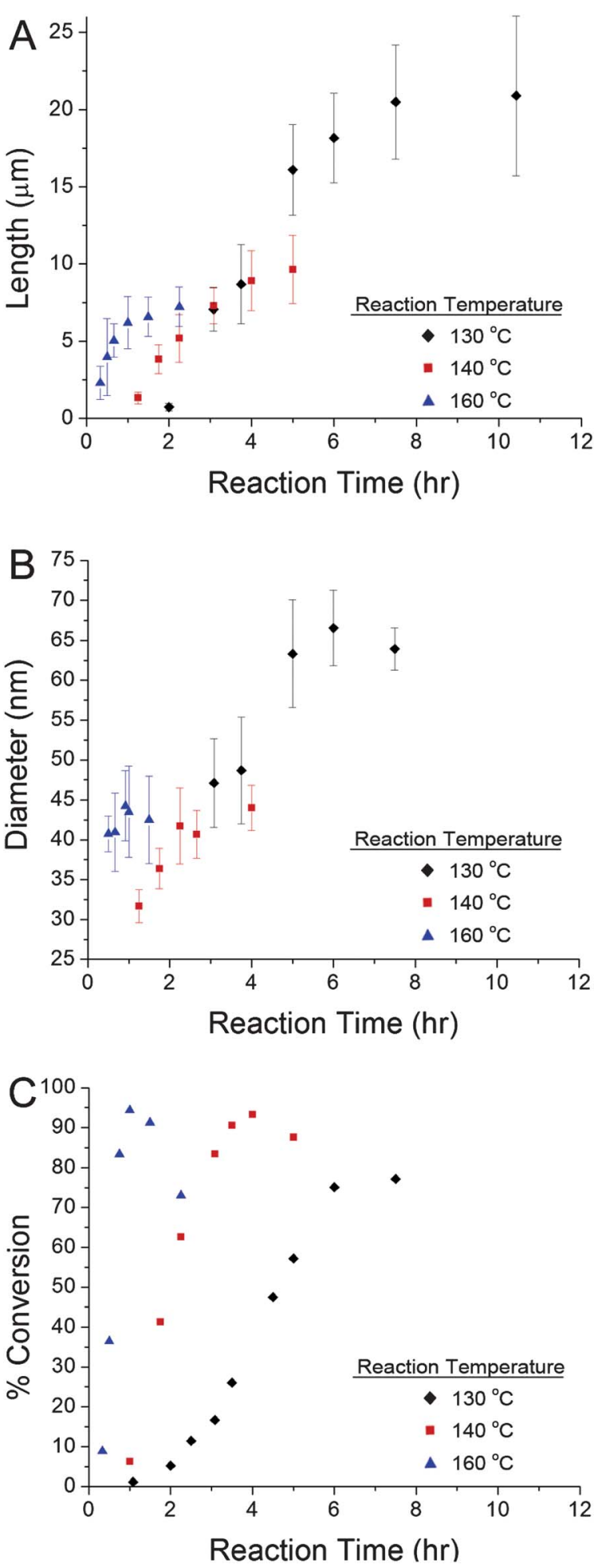

Fig. 1 (A) Silver nanowire length, (B) silver nanowire diameter, and (C) $\%$ conversion of $\mathrm{Ag}^{+}$to $\mathrm{Ag}^{0}$ vs. time for three reaction temperatures. Lower temperatures lead to the formation of longer nanowires with larger diameters.

gave off a reddish-brown gas similar in color to the NO gas given off by nitric acid; this gas had a $\mathrm{pH}=1$. If the reaction was allowed to run for long times $(>1.5 \mathrm{~h})$ at $160^{\circ} \mathrm{C}$, a large clump of silver formed at the bottom of the reaction flask. As this 
clumping did not occur as readily at $130{ }^{\circ} \mathrm{C}$, it seems likely that the aggregation of the nanowires at high temperatures and long reaction times is induced by the in situ generation of acid.

Knowing the effect of temperature and reaction time on the growth rate of the nanowires, we could judiciously select reaction conditions to produce nanowires with distinct lengths and diameters. An example set of reaction conditions for obtaining nanowires with nine different lengths and diameters is given in Fig. 2A. In some cases, the reaction procedure was modified to
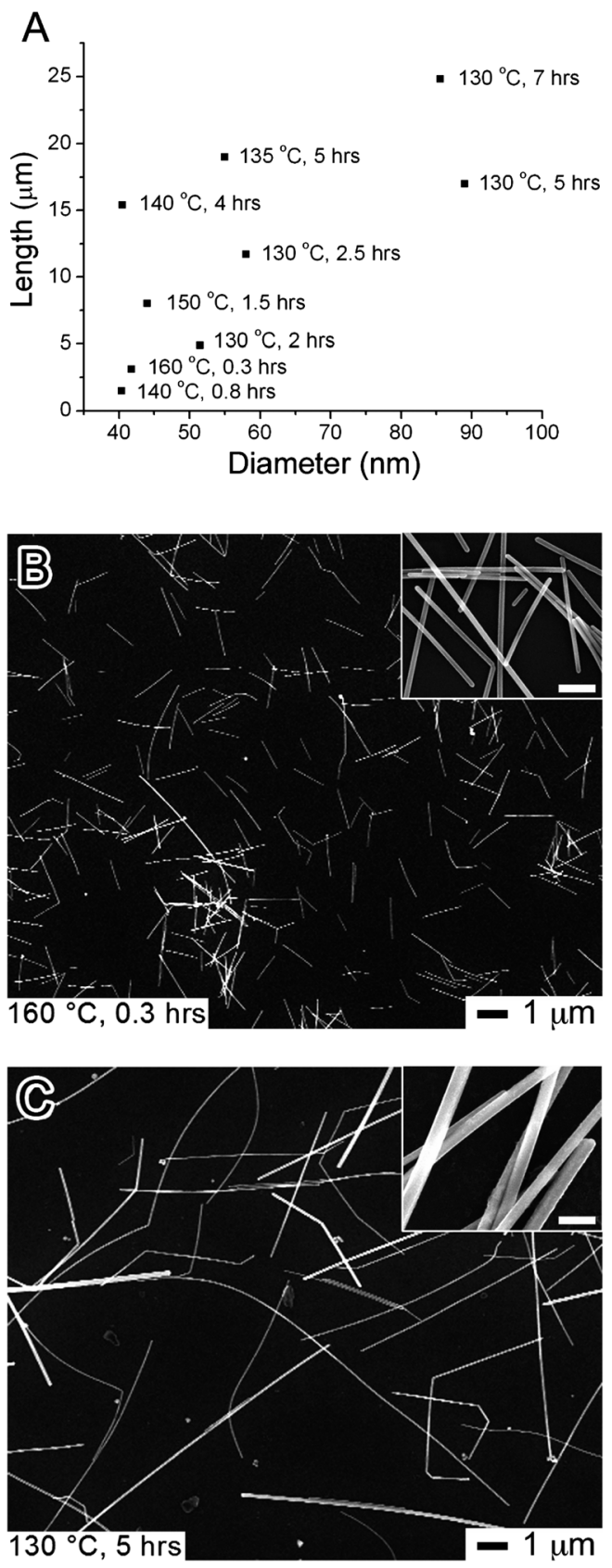

Fig. 2 (A) Reaction conditions for synthesizing nanowires with distinct lengths and diameters. (B) SEM image of silver nanowire product after growth for $0.3 \mathrm{~h}$ at $160^{\circ} \mathrm{C}$. The nanowires are $42 \pm 5 \mathrm{~nm}$ in diameter and $3 \pm 0.5 \mu \mathrm{m}$ in length. (C) SEM image of silver nanowire product after growth for $5 \mathrm{~h}$ at $130{ }^{\circ} \mathrm{C}$. The nanowires are $85 \pm 25 \mathrm{~nm}$ in diameter and $25 \pm 5 \mu \mathrm{m}$ in length. The scale bar in the inset is $200 \mathrm{~nm}$. obtain fewer nuclei, and thus nanowires with larger diameters. For example, the reaction was heated at a lower temperature (e.g. $120{ }^{\circ} \mathrm{C}$ ) during the nucleation stage of the reaction (first $5 \mathrm{~min}$ ), and then brought up to a higher temperature (e.g., $130^{\circ} \mathrm{C}$ for $7 \mathrm{~h}$ ) to induce nanowire growth. Fig. $2 \mathrm{~B} \& \mathrm{C}$ show SEM images of some of the shortest, skinniest nanowires and longest, fattest nanowires respectively. With this wide range of well-controlled nanowire morphologies in hand, we could begin to quantitatively examine the effect of nanowire length and diameter on the properties of transparent conducting films.

\section{The effect of nanowire length on the properties of transparent conducting films}

For widthless sticks in two dimensions, Pike and Seager were the first to use Monte Carlo simulations to show that, for sticks with a given length $(L)$, the critical number density of sticks required for percolation is given by eqn (2) (a value of 5.637 was

$$
N_{c} L^{2}=5.71
$$

recently proposed to be more accurate than 5.71). ${ }^{25,26}$ This equation indicates that longer nanowires will make for qualitatively better transparent conducting films because doubling the length of the nanowires decreases the number density of nanowires required for percolation by a factor of four. If more nanowires are added to a film that has just achieved percolation, more connections will be made with nanowires that were not touching the conducting path, and the conductivity of the network will increase. When the number density of nanowires $(N)$ is close to but sufficiently above $N_{c}$, the relationship between the conductivity of the film and the number density of nanowires is given by eqn (3). Here $\sigma$ can be taken as the sheet conductance of the film (the inverse of the

$$
\sigma \propto\left(N-N_{c}\right)^{t}
$$

sheet resistance), and $t$ is the conductivity exponent. $\mathrm{Li}$ and Zhang have recently shown that this relation should hold for values of $\left(N-N_{c}\right) / N_{c}$ between 0.3 and $1 .{ }^{27}$ Smaller values closer to $N_{c}$ deviate from this relation; values larger than 1 were not explored. It is generally thought that eqn (3) only holds close to percolation, but there is some ambiguity as to how far above percolation this classic equation is valid. ${ }^{28}$

The value of $t$ is typically taken as $\sim 1.3$, but $\mathrm{Li}$ and Zhang recently showed $t$ can increase from 1.2 to 1.4 as the ratio of the junction resistance $\left(R_{j}\right)$ to stick resistance $\left(R_{s t}\right)$ increases from $R_{j} /$ $R_{s t}=0.01$ to $100 .{ }^{27}$ The conductivity exponent increases with $R_{j} /$ $R_{s t}$ because adding an additional wire to a well-connected network will have a bigger impact if it shortcuts many highly resistive junctions. Relatively high experimental values of $t$ ranging from 1.46 to 1.65 have been reported for carbon nanotubes. ${ }^{29-31}$ An analogous conductivity exponent $n$ based on an approximate film thickness resulted in a high value of 1.9 for silver nanowires, and a low value of 1.0 for carbon nanotubes. ${ }^{28,32}$ We note that all these experimental values are not in the range predicted by theory; at present it is not clear why this is the case. Given the ambiguity regarding over what nanowire densities eqn (3) can be applied, as well as the value of $t$, it is clear 
that additional work in this area would be of benefit to the developing field of nanowire electrodes.

To make transparent conducting films, silver nanowires were filtered from solution onto hydrophilic, polycarbonate membranes with a pore size of $0.6 \mu \mathrm{m}$. The nanowire film was then transferred from the membrane onto a glass slide coated with clear glue in the same manner as was previously reported for copper nanowires. ${ }^{33}$ The transmittance and sheet resistance of the silver nanowire films were measured with a UV-Vis-NIR spectrometer and four-point probe, respectively.

To independently observe the effect of length on the conductivity and transmittance of silver nanowire films, we compared films composed of nanowires with the same diameter $(40 \mathrm{~nm})$ but different lengths. Fig. 3A shows that, not surprisingly, the transmittance at a given sheet resistance increased with nanowire length. Fig. 3B illustrates that as the length of the nanowires increases, the number density of nanowires necessary to achieve a given sheet resistance decreases, which results in a higher transmittance.

We plotted the experimentally determined sheet conductance $\sigma$ $v s$. the normalized number density, $N / N_{c}-1$, in Fig. $3 \mathrm{C}$, to compare the silver nanowire data to theoretical predictions. From this plot we can extract the conductivity exponent $t=1.33$. This is the first experimental conductivity exponent that agrees with theoretical predictions for 2D nanowire networks. ${ }^{27}$ Although eqn (3) has previously been suggested to be valid only near the percolation threshold, ${ }^{25,27}$ the fit to the experimental data holds remarkably well for the range of nanowire densities used in this study (up to $12 N_{c}$ ).

We note that previous authors have related the transmittance of nanowire networks to an approximate film thickness in a manner that is analogous to the method used for continuous films. ${ }^{17,32}$ This approach ignores the effect of nanowire aspect ratio on the transmittance of a nanowire film. In an attempt to more clearly link the transmittance of a nanowire film to the dimensions of the nanowires, we relate the transmittance to area coverage, $A_{c}$. The area coverage of the nanowires in a film is given by eqn (4), where $d$ is the diameter of the nanowires. Given the dimensions and

$$
A_{c}=N L d
$$

number density of the nanowires in a film, the transmittance of the film can be calculated with eqn (5). As the area coverage of nanowires in a film approaches zero, the

$$
\% T=1-a_{1} 100 A_{c}
$$

transmittance of the film approaches $100 \%$. The fitting parameter $a_{1}$ accounts for the diameter and wavelength-dependent optical properties of the nanowires, as well as the effect of the size of the open holes in the nanowire network on the transmittance of the film. A fit of eqn (5) to the data in Fig. 4A gives $\mathrm{a}_{1}=0.87$.

Fig. 4B plots sheet resistance versus area coverage to illustrate that at the same area coverage (same transmittance), films of longer wires are more conductive than films of shorter wires. For example, at an $A_{c}=0.25(\% T=79)$, the sheet resistance of films composed of nanowires $15 \mu \mathrm{m}$ in length is two orders of magnitude lower than films composed of nanowires $1.5 \mu \mathrm{m}$ in
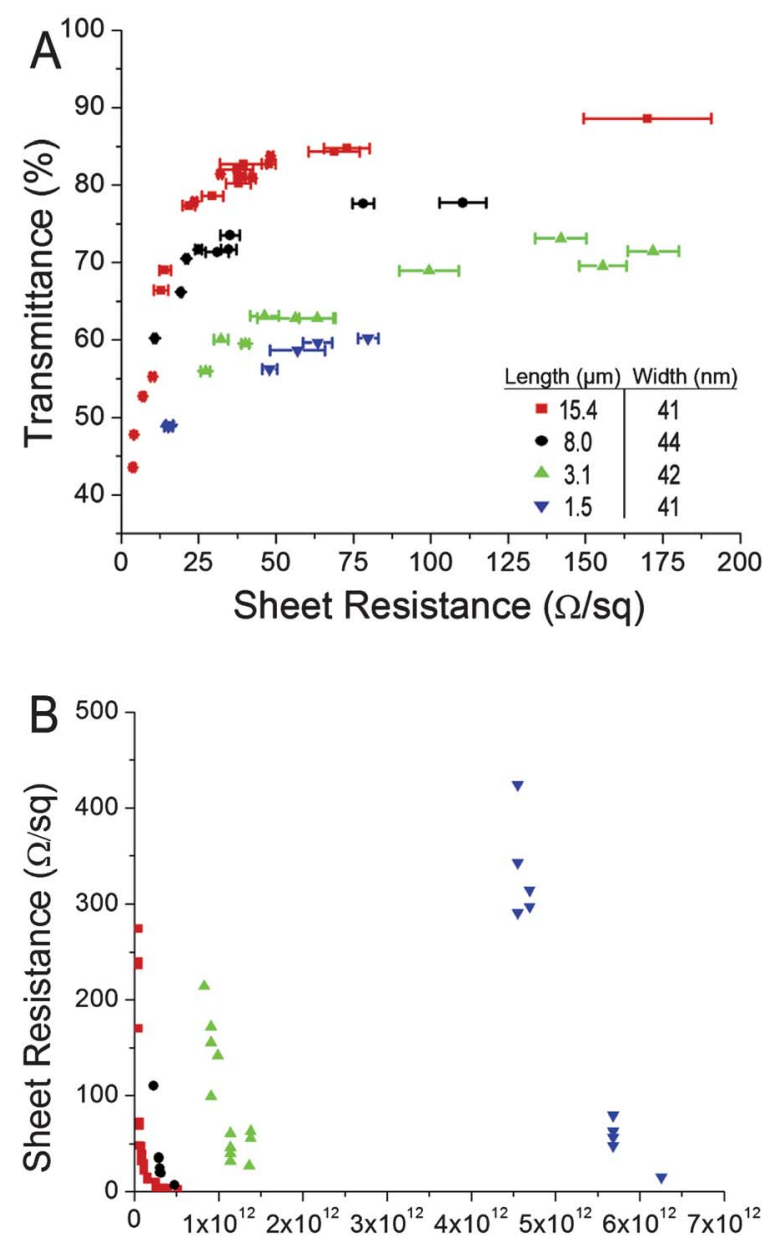

(\# wires $/ \mathrm{m}^{2}$ )

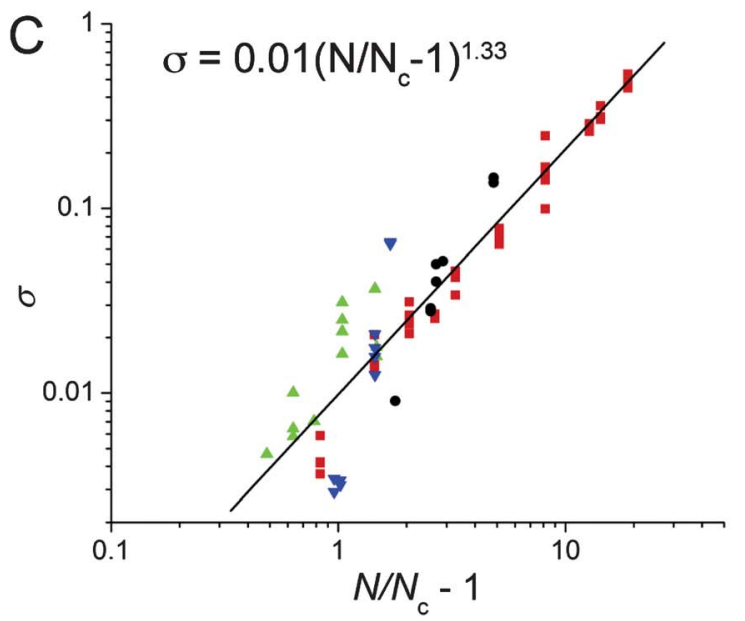

Fig. 3 (A) Transmittance (at $\lambda=550 \mathrm{~nm}$ ) vs. sheet resistance of silver nanowires with the same diameter but different lengths. (B) Plot of sheet resistance as a function of wire density illustrates the strong effect of length on the conductivity of nanowire films. (C) A logarithmic plot of sheet conductance $v s$. $N / N_{c}-1$ allows for the prediction of the sheet conductance of a nanowire film regardless of nanowire length. The solid line is a linear least squares fit to the data, and the equation for the line is shown. 

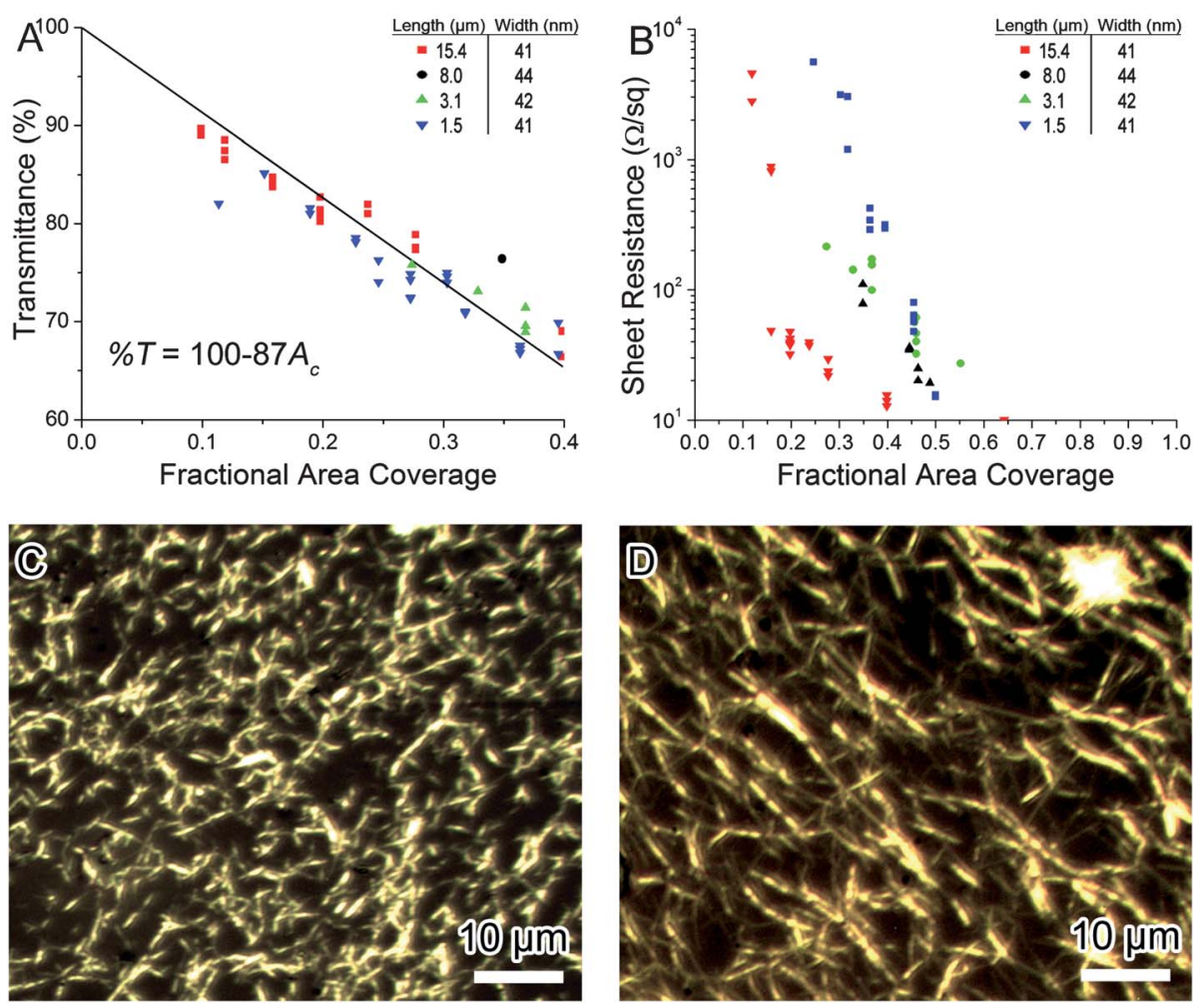

Fig. 4 (A) The transmittance $(\% T)$ of nanowire films $($ at $\lambda=550 \mathrm{~nm})$ decreases with increasing area coverage $\left(A_{c}\right)$. The solid line is a least squares fit to the data, with the equation of the line given in the lower left. (B) A plot of sheet resistance $\left(R_{s}\right) v s$. area coverage shows that longer nanowires are more conductive than shorter nanowires at the same area coverage. Dark field optical microscopy images of a film of silver nanowires (C) $1.5 \mu \mathrm{m}$ long with $A_{c}=0.4, R=290 \Omega \mathrm{sq}^{-1}$, and $\% T=67 \%$, and (D) $15.4 \mu \mathrm{m}$ long with $A_{c}=0.4, R_{s}=14 \Omega \mathrm{sq}^{-1}$, and $\% T=69 \%$, illustrates that longer nanowires overlap more at the same area coverage, resulting in a lower sheet resistance at the same transmittance.

length. This is due to the fact that, at the same area coverage, longer nanowires will overlap much more than shorter nanowires. To illustrate this visually, we compare dark-field microscope images of films composed of nanowires $1.5 \mu \mathrm{m}$ and $15 \mu \mathrm{m}$ in length at the same fractional area coverage $\left(A_{c}=0.4\right)$ in Fig. 4C \& 4D. In the film composed of nanowires $1.5 \mu \mathrm{m}$ in length, many of the nanowires were not part of the percolating network, but they nonetheless blocked light, giving this film a transmittance of $67 \%$ and a relatively high sheet resistance of $290 \Omega \mathrm{sq}^{-1}$. In contrast, at the same area coverage, and thus approximately the same transmittance $(69 \%)$, the nanowires 15 $\mu \mathrm{m}$ in length were all touching, giving this film a much lower sheet resistance of $14 \Omega \mathrm{sq}^{-1}$. These results clearly illustrate that the greater overlap enabled by longer nanowires leads to a much lower conductivity at the same transmittance.

\section{The effect of nanowire diameter on the properties of transparent conducting films}

For the sake of simplicity, we have so far ignored the effect of nanowire diameter on the properties of transparent electrodes. To begin to understand how the diameter of nanowires affects their properties in a transparent conducting film, we calculated the absorption and scattering cross-sections of individual silver nanowires for diameters between $10 \mathrm{~nm}$ and $300 \mathrm{~nm}$. The absorption and scattering cross-sections of the nanowires were calculated with the finite-difference time-domain (FDTD) method using the Meep software package. ${ }^{34}$ The experimental dielectric dispersion data of silver was used in the calculation to obtain an optical response. ${ }^{35}$ For $d \geq 200 \mathrm{~nm}$, the grid size $=$ $1 \mathrm{~nm}$; for $40 \leq d \leq 150 \mathrm{~nm}$, the grid size $=0.5 \mathrm{~nm}$, for $20 \leq d \leq$ $30 \mathrm{~nm}$, the grid size $=0.2 \mathrm{~nm}$, and for $d=10 \mathrm{~nm}$, the grid size $=$ $0.1 \mathrm{~nm}$. Two orthogonal polarization states of the normally incident plane-wave light were considered, in which the electric field was parallel or perpendicular to the cylinder axis. The nanowires were assumed to be infinitely long, and thus we report their cross-section in units of meters. To obtain the dimensionless efficiency, we divided the 1D optical cross-section by the nanowire diameter. The dimensionless absorption $\left(C_{a b s}\right)$, scattering $\left(C_{\text {scat }}\right)$ and extinction $\left(C_{e x t}=C_{a b s}+C_{s c a t}\right)$ efficiencies, averaged over the wavelength range $400-800 \mathrm{~nm}$ for both polarizations, are plotted in Fig. 5A. Due to increased scattering of light, the efficiency with which the nanowires block light increases rapidly from $3 \%$ to $147 \%$ as their diameter increases from 10 to $100 \mathrm{~nm}$. After $100 \mathrm{~nm}$, the effect of size is less dramatic and levels off as the diameter of the nanowires approaches the wavelengths of visible light.

Perhaps because of the strong effect of nanowire diameter on their scattering efficiency, it has often been hypothesized that nanowire networks with smaller diameters will transmit more light at a given sheet resistance than films of nanowires with larger diameters. ${ }^{17,36}$ We have tried to gain some insight into whether this is in fact true by plotting the conductance to extinction ratio, $\sigma_{D C} A / \sigma_{e x t}$, vs. nanowire diameter in Fig. 5B. 

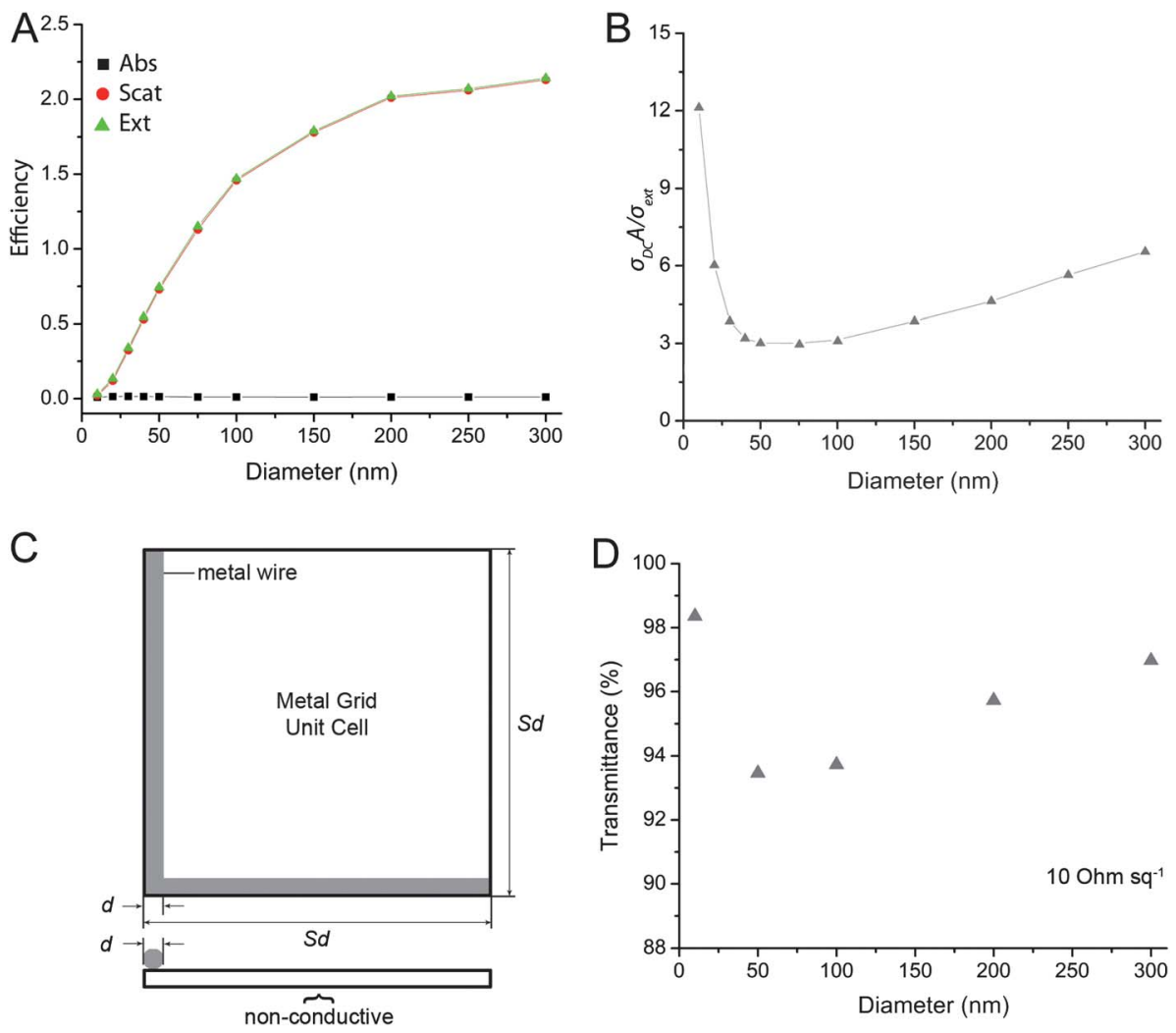

Fig. 5 (A) FDTD calculations of the absorption, scattering, and extinction efficiency of silver nanowires $v s$. diameter, averaged over 400-800 nm. (B) A plot of the conductance to extinction ratio for silver nanowires $v$ s. diameter. (C) Schematic of a nanowire grid unit cell. (D) Plot of transmittance at a sheet resistance of $10 \Omega \mathrm{sq}^{-1}$ vs. nanowire diameter for nanowire grid unit cells.

Here $\sigma_{D C}$ is the DC conductivity of the metal adjusted for nanowire size using Sambles's equation 13 in ref. $37 . .^{37} \mathrm{We}$ assumed a surface roughness $h=0.03 \mathrm{~nm}$ (atomically smooth), which seems to be justified given the recent results of Critchley et al. ${ }^{37,38} A$ is the cross-sectional area of the nanowire, and $\sigma_{e x t}$ is the extinction cross-section of the nanowires.

Fig. 5B indicates that, with the exception of nanowires with diameters smaller than $20 \mathrm{~nm}$, large-diameter nanowires will have higher conductance to extinction ratios than nanowires with smaller diameters. This makes sense because the amount of light blocked by large wires roughly scales with diameter $d$, whereas the conductance scales with $d^{2}$. Thus, based on the properties of individual nanowires, we can tentatively conclude that nanowire networks with smaller diameters will provide higher transmittances at a given sheet resistance, but only for nanowires smaller than $20 \mathrm{~nm}$. To date there are no reports of transparent conductive networks of silver nanowires with diameters smaller than $20 \mathrm{~nm}$.

To facilitate comparison with experimental results, we have extended these calculations on individual nanowires to metal grids. Fig. 5C shows a picture of the unit cell of a metal grid, which is defined by a nanowire of diameter $d$, and a lattice parameter $S d$. The $\% T$ of this unit cell can be calculated with eqn (6). Here $\sigma_{\text {ext }}$ is the

$$
\% T=\left(1-\frac{2 \sigma_{e x t}}{S}+\frac{\sigma_{e x t}^{2}}{S^{2}}\right) \times 100
$$

extinction cross-section of a nanowire of a given diameter. For a given sheet resistance $\left(R_{S}\right), S$ can be calculated by eqn (7). Here again, $\sigma_{D C}$ is the DC conductivity of the

$$
S=\frac{\pi(d / 2)^{2} R_{s} \sigma_{D C}}{d}
$$

metal adjusted for nanowire size. Fig. 5D plots $\% T v s$. $d$ for nanowire grid unit cells with $S$ adjusted to give $R_{s}=10 \Omega \mathrm{sq}^{-1}$ regardless of the nanowire diameter. The general trend matches that of Fig. 5B in that the transmittance of the silver nanowire grids improves only for the smallest nanowire diameter of $10 \mathrm{~nm}$. These calculated results correlate well to literature data. Metal grids consisting of lines $10-\mu \mathrm{m}$-wide exhibit sheet resistances of $10 \Omega \mathrm{sq}^{-1}$ at a transmittance of $95 \%{ }^{39}$ Silver nanowires with a diameter of $\sim 60 \mathrm{~nm}$ exhibited a transmittance of less than $90 \%$ at the same sheet resistance of $10 \Omega \mathrm{sq}^{-1} \cdot{ }^{16}$ Based on these calculated results and literature data, one might conclude that decreasing nanowire diameter does not always improve the performance of a nanowire network; it depends on the size to which the nanowire diameter is reduced.

We note that in calculating the transmission of light through metal grids, we have ignored the size-effect of the holes. The relationship between the diameter $(d)$ and the width of the open hole $(S d-d)$ for a metal grid with a sheet resistance of $10 \Omega \mathrm{sq}^{-1}$ can be calculated with eqn (8). Ignoring the size-effect of holes on the transmission of light

$$
(S d-d)=0.35 d^{2}
$$


is likely a good approximation for nanowires $50 \mathrm{~nm}$-in-diameter or larger, for which the hole is $>1.1 \mu \mathrm{m}$ in width. However, for nanowire diameters $<40 \mathrm{~nm}$, the hole size is smaller than the wavelengths of visible light, and there will likely be an additional effect of the hole size on transmittance.

To begin to understand the influence of nanowire diameter and hole size on the properties of nanowire networks, we used a 3D FDTD calculation to determine the transmittance $(\lambda=550 \mathrm{~nm})$ of random networks of nanowires of the same length $(10 \mu \mathrm{m})$, but different diameters and area coverages. The simulated area was $10 \times 10 \mu \mathrm{m}$ for nanowires $300 \mathrm{~nm}$ in diameter, and $5 \times 5 \mu \mathrm{m}$ for the other diameters. The grid size was $2 \mathrm{~nm}$ for the $30-\mathrm{nm}$ nanowires, $4 \mathrm{~nm}$ for the $50-\mathrm{nm}$-nanowires, $4 \mathrm{~nm}$ for the $100-\mathrm{nm}$ nanowires, and $7 \mathrm{~nm}$ for the $300-\mathrm{nm}$-nanowires. Due to the random variation in the number of wires in the simulated area, each simulation was performed three times. The data points plotted in Fig. 6A give the average value of these three simulations, and the error bars give the full range of the calculated values ([high - low]/2). We note that this is the first time the calculated transmission of random nanowire networks has been reported. Similar to the experimental data in Fig. 4A, the calculated transmittance is linearly dependent on area coverage. For comparison, we plotted $\left(1-A_{c} \sigma_{e x t}\right) \times 100 v s$. area fraction in Fig. 6B. This plot gives the percentage of light that would be transmitted for a given area coverage of nanowires of a given diameter if the effects of hole size and nanowire overlap are ignored.

There are two contrasting effects that can be illustrated by comparing the data in Fig. 6A \& B. One is that the calculated transmittance of nanowire networks consisting of nanowires greater than $100 \mathrm{~nm}$ in diameter is higher than is obtained simply by multiplying the area coverage by the extinction efficiency. For example, at $A_{c}=0.5$, the transmittance of a random nanowire network made of nanowires $300 \mathrm{~nm}$ in diameter is $61 \%$, whereas $\left(1-A_{c} \sigma_{e x t}\right) \times 100=-6 \%$. This comparison illustrates that it is important to take into account the overlapping extinction crosssections of nanowires (especially larger nanowires) in order to obtain an accurate estimate of the transmittance of a random nanowire network.

The second effect is the dependence of the transmittance on the size of the holes in the nanowire network. The effect of hole size is perhaps best illustrated by noting that, in contrast to the $300-\mathrm{nm}$ nanowire-networks, networks composed of nanowires $30 \mathrm{~nm}$ in diameter have lower transmittances (by about 6\%) than is obtained by multiplying the area coverage by the extinction efficiency. This is in part due to the fact that, as illustrated by Fig. $6 \mathrm{C}-\mathrm{F}$, the size of the holes in the nanowire network at a given area coverage decreases with decreasing nanowire diameter. In addition, the extinction efficiency $\left(C_{\text {ext }}\right)$ of the 30$\mathrm{nm}$-nanowires is 0.28 , while that of the $300-\mathrm{nm}$-nanowires is 2.1 . Thus, at small nanowire diameters, the effect of hole size on the transmittance becomes more dominant than the effect of nanowire overlap.

Finally, the images of nanowire networks in Fig. 6C-F illustrate the important effect of nanowire diameter on network connectivity and thus conductivity. For a given area fraction and nanowire length, decreasing diameter increases the number of nanowires. Increasing the number of nanowires increases the number of connections between the nanowires, and, as given by eqn (3), increases the conductivity of the nanowire network. This effect of nanowire diameter on connectivity is a critical difference between solution-coated, random nanowire networks and metal grids. The effect of diameter on number density is especially important at high transmittance (low area coverage), at which point there are relatively few connections between nanowires.
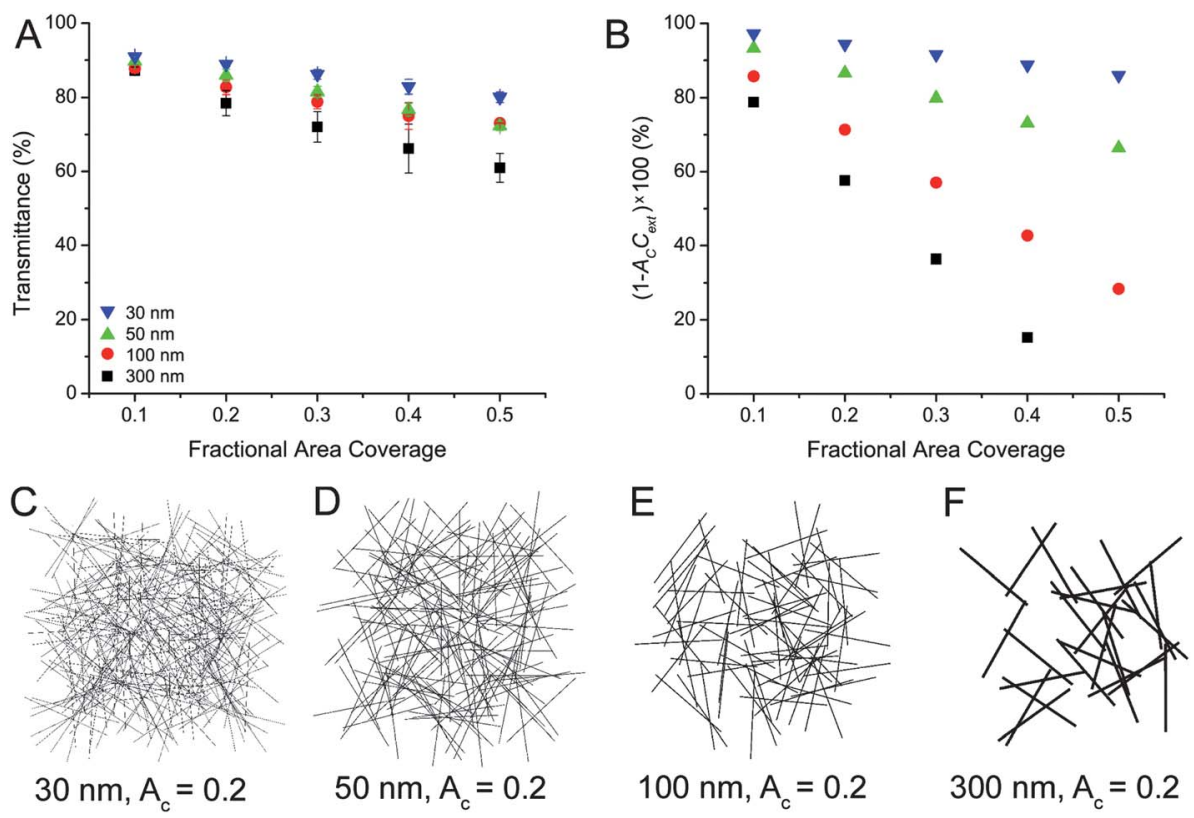

Fig. 6 (A) Calculated transmittance (at $\lambda=550 \mathrm{~nm}$ ) vs. area coverage for random networks of silver nanowires with different diameters. (B) Plot of the light transmitted through a given area coverage of nanowires (at $\lambda=550 \mathrm{~nm}$ ) if the effect of hole size and overlap of extinction cross-sections is ignored. (C) Example pictures of the networks for which the transmittance is plotted in (A) show the effect of nanowire diameter on hole size and connectivity. 
To better quantify the effect of nanowire diameter on network connectivity, we again used a 3D FDTD simulation to determine the transmittance of nanowire networks at the critical number density $\left(N_{c}\right)$ required for percolation (Fig. 7). As the lengths of the nanowires in the calculation are the same $(10 \mu \mathrm{m})$, the number density of nanowires is constant, and thus the area coverage of nanowires (i.e., the amount of light blocked) will increase with increasing diameter. This results in a transmittance of $92 \%$ for the $30-\mathrm{nm}$-nanowires, but only $81 \%$ for the $300-\mathrm{nm}$ nanowires. These values set the maximum transmittance that can be obtained for these nanowire networks and still have a conducting path.

To summarize, our calculations indicate there are two opposing effects of nanowire diameter on the properties of nanowire networks: (1) increasing nanowire diameter increases the conductance to extinction ratio (except for silver nanowires with diameters $<20 \mathrm{~nm}$ ), (2) networks consisting of nanowires with smaller diameters will exhibit more connectivity at a given area coverage. Given these opposing effects (and ignoring nanowires $<20 \mathrm{~nm}$ ), we might expect networks composed of nanowires with smaller diameters to outperform networks composed of nanowires with larger diameters, but only at low area coverages (high transmittance), at which point the number of connections between nanowires limits the sheet resistance of the network. Conversely, at high area coverages, when all the nanowires in the network are connected, we would expect the conductance to extinction ratio to dominate, and nanowires with larger diameters will give lower sheet resistances.

Experimental data plotted in Fig. 8 conforms to these general conclusions. The transmittance of films composed of nanowires $15 \mu \mathrm{m}$ long and $41 \mathrm{~nm}$ in diameter is about $5 \%$ greater than films composed of nanowires with diameters twice as large $(85 \mathrm{~nm})$, but only at relatively high sheet resistances. At the highest sheet resistance plotted (175 $\Omega \mathrm{sq}^{-1}$ ), films of smaller diameter nanowires (41 nm by $15 \mu \mathrm{m})$ can even overcome a deficit in length of $10 \mu \mathrm{m}$ to match the sheet resistance of films composed of largerdiameter nanowires ( $85 \mathrm{~nm}$ by $25 \mu \mathrm{m}$ ). In contrast, at low sheet resistances, films of nanowires with larger diameters slightly outperform films of nanowires with smaller diameters.

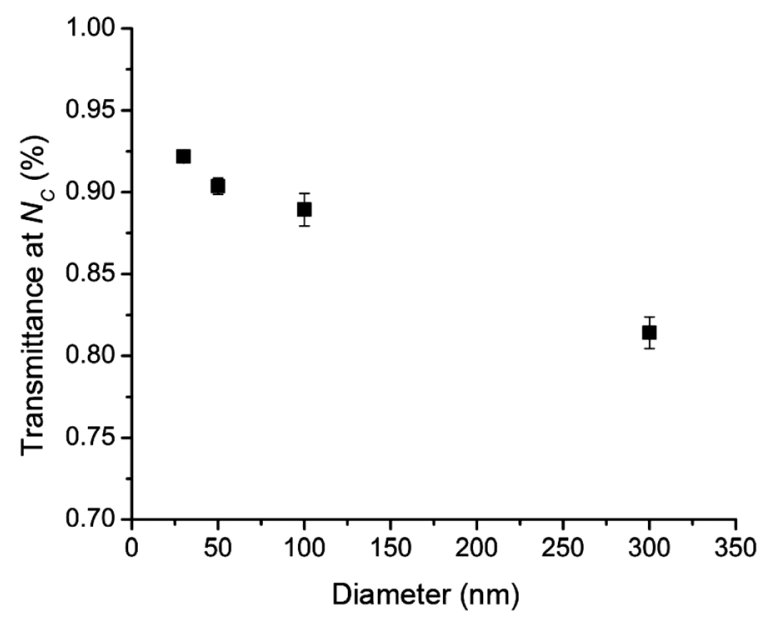

Fig. 7 The transmittance of a random network of $10-\mu \mathrm{m}$-long nanowires at the critical number density $\left(N_{c}\right)$ required for percolation.

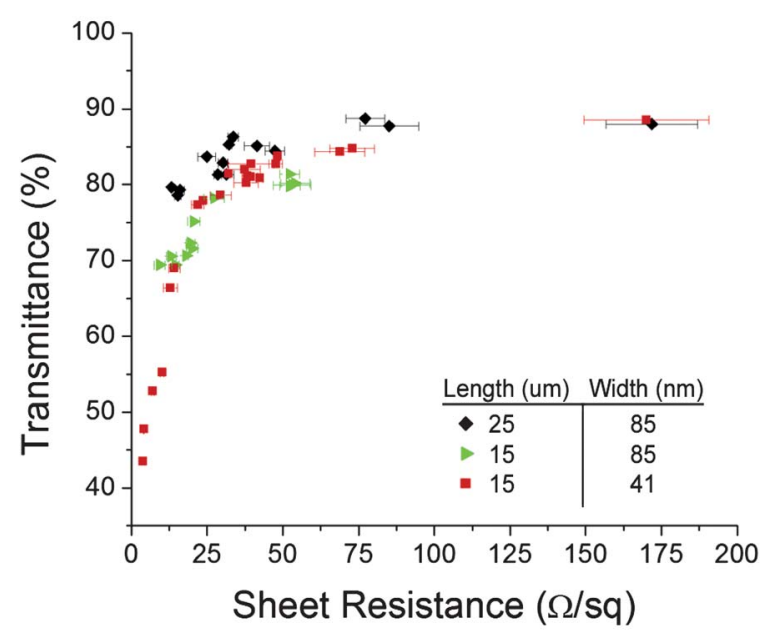

Fig. 8 Transmittance (at $\lambda=550 \mathrm{~nm}$ ) vs. sheet resistance of silver nanowires with different diameters. Nanowires with smaller diameters outperform nanowires with larger diameters at high transmittance, but at low transmittance the opposite is true.

\section{Conclusions}

We utilized the temperature-dependent reducing power of ethylene glycol to control the assembly of silver atoms into nanowires with independently controlled lengths and diameters. By comparing films composed of nanowires with different lengths but the same diameter, we tested percolation theory relations for the effect of nanowire density and length on the sheet conductance of a nanowire network, and obtained a value of 1.33 for the conductivity exponent. This is the first experimentally determined conductivity exponent for a $2 \mathrm{D}$ nanowire network that falls in the range of values predicted by theory. We also showed that the transmittance of a network composed of nanowires of a given diameter is linearly dependent on area coverage, and is not dependent on nanowire length. Finally, we presented calculations and experimental data that shows thinner nanowires do not always make better transparent electrodes. Decreasing nanowire diameter improves optoelectronic performance only for nanowires less than $20 \mathrm{~nm}$ in diameter, or for high-transmittance networks in which the number of connections between nanowires limits the conductivity of the network. For networks in which the nanowires are highly connected, nanowires with larger diameters will outperform nanowires with smaller diameters due to their larger conductance to extinction ratios. Nanowires less than $20 \mathrm{~nm}$ in diameter are the exception to this trend because they scatter very little light.

After reading this article, we hope the reader comes away with a deeper understanding of the complexities involved in predicting the structure-property relationships of nanowire networks. The transmittance of a nanowire network depends not only on the optical properties of individual nanowires, but also on the random overlap of their extinction cross-sections, and the random size of the holes in the network. Similarly, calculating the conductance of a nanowire network is complicated by the effect of diameter on the conductance of individual nanowires, and the effect of diameter and length on network connectivity. Accounting for all these factors will require additional 
experimental and theoretical work, in particular the synthesis of a wider range of nanowire diameters, and the development of a model to relate the conductivity of the nanowire network to nanowire diameter. Such work will provide a rational basis for the design of nanowire network architectures for displays, organic solar cells, and organic light emitting diodes.

\section{Acknowledgements}

B.J.W. and P.C. acknowledge startup funding from Duke University, and NSF's Research Triangle MRSEC (DMR1121107). A.R.R. was supported by a fellowship under the Duke Nanoscience Certificate Program, and the NSF's Research Triangle MRSEC. S.M.B. acknowledges the support of a grant from Duke's Undergraduate Research Support Office. Z.Y.L. was supported by the National Key Basic Research Special Foundation of China under Grant Nos. 2011CB922002 and the National Natural Science Foundation of China under Grant Nos. 60736041 and 10874238.

\section{References}

1 R. G. Gordon, MRS Bull., 2000, 25, 52.

2 U.S. Geological Survey, Mineral Commodity Summaries. Indium, 2009, p. 76.

3 J. A. Rogers, T. Someya and Y. G. Huang, Science, 2010, 327, 1603.

4 J. Y. Lee, S. T. Connor, Y. Cui and P. Peumans, Nano Lett., 2008, 8, 689.

5 W. Gaynor, J. Y. Lee and P. Peumans, ACS Nano, 2009, 4, 30.

6 X. Y. Zeng, Q. K. Zhang, R. M. Yu and C. Z. Lu, Adv. Mater., 2010, 22, 4484.

7 T. Sekitani, H. Nakajima, H. Maeda, T. Fukushima, T. Aida, K. Hata and T. Someya, Nat. Mater., 2009, 8, 494.

8 X. N. Ho, L. N. Ye, S. V. Rotkin, Q. Cao, S. Unarunotai, S. Salamat, M. A. Alam and J. A. Rogers, Nano Lett., 2010, 10, 499.

9 S. C. B. Mannsfeld, B. C. K. Tee, R. M. Stoltenberg, C. Chen, S. Barman, B. V. O. Muir, A. N. Sokolov, C. Reese and Z. N. Bao, Nat. Mater., 2010, 9, 859.

10 D. S. Hecht, L. B. Hu and G. Irvin, Adv. Mater., 2011, 23, 1482.

11 L. B. Hu, H. Wu and Y. Cui, MRS Bull., 2011, 36, 760.

12 C. M. Niu, MRS Bull., 2011, 36, 766.
13 E. N. Dattoli and W. Lu, MRS Bull., 2011, 36, 782.

14 A. Elschner and W. Lovenich, MRS Bull., 2011, 36, 794.

15 S. Kirchmeyer and K. Reuter, J. Mater. Chem., 2005, 15, 2077.

16 D. S. Leem, A. Edwards, M. Faist, J. Nelson, D. D. C. Bradley and J. C. de Mello, Adv. Mater., 2011, 23, 4371.

17 S. De, T. M. Higgins, P. E. Lyons, E. M. Doherty, P. N. Nirmalraj, W. J. Blau, J. J. Boland and J. N. Coleman, ACS Nano, 2009, 3, 1767.

18 L. B. Hu, H. S. Kim, J. Y. Lee, P. Peumans and Y. Cui, ACS Nano, 2010, 4, 2955.

19 U.S. Geological Survey, Mineral Commodity Summaries. Indium, 2012, p. 74.

20 U.S. Geological Survey, Mineral Commodity Summaries. Silver, 2012, p. 146.

21 B. Wiley, Y. G. Sun and Y. N. Xia, Langmuir, 2005, 21, 8077.

22 S. E. Skrabalak, B. J. Wiley, M. Kim, E. V. Formo and Y. Xia, Nano Lett., 2008, 8, 2077.

23 Y. G. Sun, B. Mayers, T. Herricks and Y. N. Xia, Nano Lett., 2003, 3, 955.

24 B. Wiley, Y. Sun and Y. Xia, Acc. Chem. Res., 2007, 40, 1067.

25 G. E. Pike and C. H. Seager, Phys. Rev. B: Solid State, 1974, 10, 1421.

26 J. T. Li and S. L. Zhang, Phys. Rev. E: Stat., Nonlinear, Soft Matter Phys., 2009, 80, 040104.

27 J. T. Li and S. L. Zhang, Phys. Rev. E: Stat., Nonlinear, Soft Matter Phys., 2010, 81, 021120.

28 S. De and J. N. Coleman, MRS Bull., 2011, 36, 774.

29 L. Hu, D. S. Hecht and G. Gruner, Nano Lett., 2004, 4, 2513.

30 D. Hecht, L. B. Hu and G. Gruner, Appl. Phys. Lett., 2006, 89, 133112.

31 H. E. Unalan, G. Fanchini, A. Kanwal, A. Du Pasquier and M. Chhowalla, Nano Lett., 2006, 6, 677.

32 S. De, P. J. King, P. E. Lyons, U. Khan and J. N. Coleman, ACS Nano, 2010, 4, 7064.

33 A. R. Rathmell, S. M. Bergin, Y.-L. Hua, Z.-Y. Li and B. J. Wiley, Adv. Mater., 2010, 22, 3558.

34 A. F. Oskooi, D. Roundy, M. Ibanescu, P. Bermel, J. D. Joannopoulos and S. G. Johnson, Comput. Phys. Commun., 2010, 181, 687.

35 P. B. Johnson and R. W. Christy, Phys. Rev. B: Solid State, 1972, 6, 4370.

36 Y. C. Lu and K. S. Chou, Nanotechnology, 2010, 21, 215707.

37 J. R. Sambles, K. C. Elsom and T. W. Preist, J. Phys. F: Met. Phys., 1982, 12, 1169.

38 K. Critchley, B. P. Khanal, M. L. Gorzny, L. Vigderman, S. D. Evans, E. R. Zubarev and N. A. Kotov, Adv. Mater., 2010, 22, 2338.

39 J. Woerle and H. Rost, MRS Bull., 2011, 36, 789. 